QUARTERLY OF APPLIED MATHEMATICS

VOLUME LXVIII, NUMBER 1

MARCH 2010, PAGES 91-112

S 0033-569X(09)01177-9

Article electronically published on October 21, 2009

\title{
ASYMPTOTICS OF SURFACE WAVES OVER RANDOM BATHYMETRY
}

\author{
$\mathrm{BY}$ \\ WALTER CRAIG (Department of Mathematics, McMaster University, Hamilton, Ontario L8S $4 K 1$, \\ Canada) \\ AND \\ CATHERINE SULEM (Department of Mathematics, University of Toronto, Toronto, Ontario M5S \\ 3G3, Canada)
}

In honor of Walter Strauss on the occasion of his seventieth birthday

\begin{abstract}
This paper addresses the propagation of free surface water waves over a variable seabed in the long wavelength scaling regime. We consider the situation in which the bathymetry is given by a stationary random process which has a correlation length substantially shorter than the wavelength of the principal surface wave components. An asymptotic description shows that the water waves problem is modeled by an effective system of equations that is related to the $\mathrm{KdV}$, however in a reference frame given in terms of random characteristic coordinates, and in addition with a random amplitude modulation and random scattered component. The resulting random processes are strongly correlated and have canonical limits due to the Donsker invariance principle. Our analysis is based on the Hamiltonian description of water waves and long wave perturbation theory and a new criterion for asymptotic expansions of partial differential equations with rapidly varying coefficients. In this paper we give a detailed analysis of the transformation to random characteristic coordinates and the asymptotic form of the resulting transformed partial differential equations. A companion paper (de Bouard, A., Craig, W., Díaz-Espinosa, O., Guyenne, P., Sulem, C., Long wave expansions for water waves over random topography, Nonlinearity 21 (2008), 2143-2178) analyses in detail the asymptotic behavior of the resulting expression for solutions, and their consistency with the derivation of the effective model equations.
\end{abstract}

Received December 31, 2008.

2000 Mathematics Subject Classification. Primary 76B15.

The first author was partially supported by the Canada Research Chairs Program and NSERC grant \#238452-06. The second author was partially supported by NSERC grant \#46179-05.

E-mail address: craig@math.mcmaster.ca

E-mail address: sulem@math.toronto.edu

(C)2009 Brown University Reverts to public domain 28 years from publication 
1. Introduction. In this paper we present recent results concerning the asymptotic description of surface waves in the long wave regime over a variable bottom, extending and elaborating upon [3. The underlying assumption is that the bottom topography is modeled by a random process varying on short spatial scales. This is an important physical setting of nonlinear waves propagating in a random environment, modeling the situation where the bathymetry is known on a long length scale, but where the details of the bottom variations are unknown. In this setting it is natural to treat functions describing bathymetric variations as random elements of a statistical ensemble. The question of asymptotic behavior is that of homogenization, and in contrast to this, of the persistence of random realization-dependent effects in the solution. Compared to the random case, the problem of a periodic bottom is simpler ([13, [6], 9]), and homogenizes fully due to a strong scale separation lemma.

In the case of constant depth, it is a well-known result that linear surface waves decay algebraically in time due to dispersive effects. In contrast, nonlinear wave evolution exhibits solutions which propagate in time coherently, and which efficiently transport momentum and energy over large distances. When there is bottom topography present, there are the additional effects of modulation and Bragg scattering that influence solutions. In particular, fluid domain bathymetry given by a stationary random process has a localizing effect due to random Bragg scattering, a linear phenomenon known from the theory of random Schrödinger operators as Anderson localization. It is thus a principal issue, whether nonlinear surface water waves are effectively localized by the effects of random scattering, or does the nonlinear character of the evolution persist. In particular, it is an important question whether coherent overall features of the wave motion remain which allow for coherent time evolution.

In the fluid dynamical problem of water waves, the bathymetry is fixed but is considered an unknown realization, and it is important to understand both the averaged (that is, homogenized) as well as the realization-dependent features of the solution. In the chosen scaling regime, there are homogenized effects which modify the effective wavespeed, and possibly the stability, of wave propagation. In addition, the bathymetry also contributes random, realization-dependent effects that are as important or more so as the nonlinearity and the dispersion of the problem. These influence the solution in three principal ways: (1) they introduce a random phase to the propagation so that, for example, the solution undergoes random fluctuations in its location; (2) the solution amplitude is modulated by a random factor; (3) there is a random scattered component to the evolution of the solution. Despite these effects, we find that solutions still retain their nonlinear character, in that they evolve in a coherent fashion, once the random phase and modulation is taken into account, and they persist in their ability to propagate energy and momentum over large distances, at least as long as allowed within the domain of validity of the scaling regime. Due to the realization-dependent aspects of wave propagation, this paper concentrates on constructing individual solutions for each particular realization of the bathymetry. Quantities such as the mean value of the solution, or its variance (or even higher moments) are taken as statistical predictions of the behavior of solutions, but should be considered separately from the individual solutions themselves. 
In particular this issue arises in considering the question of diffusion. One expects for example the mean value to exhibit spatial diffusive effects, while the solution itself satisfies a conservative equation for each given realization of the ensemble. A detailed description of long wave scaling regimes in the presence of randomness, and the order of the appearance of diffusion in the evolution problem, is given in [3].

Our interest in this problem stems from the paper of Rosales \& Papanicolaou (13] (1983)), who considered the KdV scaling regime of the water waves problem in a variable fluid domain, with both a rapidly varying periodic bottom and a random bottom. Their results are that the periodic case homogenizes fully, while in the random case they derive the first term in an asymptotic description of the solution. In their treatment of the random problem they considered bottom variations generated by stationary processes of the form $\beta(x, \omega)=\partial_{x} \mu(x, \omega)$, which has the property that $\sigma_{\beta}=0$. One of our main results is to extend their description to the case $\sigma_{\beta}>0$. There has been recently renewed interest in the subject of random bathymetry. The paper of Mei \& Li ([12] (2004)) considers the Boussinesq and the KdV scaling regimes of the water waves problem, in a situation in which bottom variations are of the same typical wavelength as the surface waves, and in Mei \& Hancock (11] (2003)) the modulational scaling regime is considered. The recent paper of Garnier, Muñoz Grajales \& Nachbin [10] is closest to our own considerations. Starting from a variable coefficient Boussinesq equation model of the problem of water waves, and introducing a random time variable, they derive the principal component of the solution in the analogous KdV regime. They furthermore show that this solution undergoes random phase variations, and that the asymptotic character of the phase variations is a Brownian motion. In this context, our paper (3] (2008)) and the present article derive the phase and amplitude variations of the solution in the $\mathrm{KdV}$ scaling regime and give an expression for the scattered component of the solution. We furthermore verify that the expression in [10] for the random wavespeed of their Boussinesq model corresponds up to $o(\varepsilon)$ with the value derived from the full Euler equations, while noting that in both cases the nonlinearity enters at order $O\left(\varepsilon^{2}\right)$.

In this work, we consider the class of bottom variations given by a stationary ergodic process $\beta$ which is sufficiently mixing so that the central limit theorem holds, in the form of the Donsker invariance principle for functionals. For such processes, the variance $\sigma_{\beta}^{2}$ is well defined, and we consider the generic situation in which $\sigma_{\beta} \neq 0$. Furthermore, we require that $\beta \in L^{\infty}(\mathbb{R})$ for P-a.e. realization, in order that an appropriate class of probabilistic limit theorems holds.

The principal results of this paper are the derivation, solution and justification of a system of effective equations which model the equations of free surface water waves. The model system has the form

$$
\begin{aligned}
\partial_{t} r & =-\partial_{X}\left(c_{\varepsilon}(X) r+\varepsilon^{2}\left(c_{1} \partial_{X}^{2} r+\frac{3}{2} c_{2} r^{2}\right)\right)+\varepsilon^{2} b r, \\
\partial_{t} s & =\partial_{X} s+\frac{\varepsilon^{-3 / 2}}{4} \partial_{x} \gamma\left(\frac{X}{\varepsilon}\right) r,
\end{aligned}
$$

in which the parameters $c_{1}, c_{2}$ are fixed constants. The coefficient of the acceleration of gravity and the average depth have been normalized to 1 , while $c_{\varepsilon}(X)=\left(1-\frac{\varepsilon}{2} \gamma(X / \varepsilon)-\right.$ $\left.\varepsilon^{2} a\right)$ is a variable coefficient that plays the role of a corrected linear wave speed, where 
$\gamma=\operatorname{sech}\left(D_{x}\right) \beta$ is given in terms of the stationary ergodic process $\beta(\cdot, \omega)$. In addition, there are two free parameters $a$ and $b$. In the analysis, these parameters are determined by the criterion that the solutions of (1.1), (1.2) are consistent with this regime of approximation of the free surface water wave equations. Furthermore, the forcing term $\varepsilon^{-3 / 2} \partial_{x} \gamma r$ of (1.2) is controlled by a probabilistic analysis.

Our asymptotic analysis of the Euler equations in the long wave regime, in the presence of short spatial scale oscillations of the bottom, is at heart a formal asymptotic expansion, for which some aspects of the derivation have a mathematically rigorous justification. However others, and in particular the truncation of the Hamiltonian, have not yet been fully justified with rigorous mathematical analysis.

Introducing random characteristic coordinates, solutions of equations (1.1) are described asymptotically by solutions of the deterministic equation

$$
\partial_{\tau} q=-c_{1} \partial_{Y}^{3} q-3 c_{2} q \partial_{Y} q+b q,
$$

which is a version of the KdV. Inverting the transformation from characteristic coordinates, solutions $r(X, t)$ are asymptotic to solutions of the original equation (1.1), at least in a distributional sense. In turn, we show that the asymptotic solutions $(r(X, t), s(X, t))$ to the model equations are actual asymptotic solutions of the water wave problem when the two free parameters $(a, b)$ are chosen consistently. Namely, $(a, b)$ are selected so that the asymptotic behavior of the resulting solution is consistent with the elimination of higher order terms in the derivation of the model system (1.1), (1.2).

In light of the Donsker theorem, we find that the characteristic trajectories are of the form of straight lines modified by a Brownian motion. This implies that the phase of $r(X, t)$ undergoes Brownian motion in physical variables. Furthermore, the amplitude is subject to random modulation in the form of white noise, and the scattering component of the solution is also quantified as a superposition of related singular processes.

The model equation (1.1) differs from the usual KdV due to the presence of the additional linear term $\varepsilon^{2} b r$ which arises in the consistency analysis. In many cases, for example if the statistics of the bottom are reversible, one has $b=0$. In the general case however, the coefficient $b$ will be nonzero due to skewness of the statistics of $\beta$, in which case its sign is important to the stability and the properties of attenuation or growth of solutions.

The paper is organized as follows. In section 2 we present the probabilistic theorems and scale separation lemmas and set up their applications to the derivation of the limiting expression of the solution. In section 3 we describe the criterion under which our asymptotic analysis is specified. We subsequently derive the effective system of equations to the water wave problem in the long wave scaling; these turn out to be coupled KdV equations with random, rapidly oscillating coefficients. In section 4 we analyze the system of random characteristic coordinates and derive solutions of the system (1.1), (1.2) perturbatively. Section 5 is devoted to the consistency analysis. From the expressions for the solutions we construct, we compute a posteriori all terms that have been neglected in the asymptotic equations and prove that they can be accommodated through adjustment of the two free parameters in the effective system. Sections 4 and 5 use the limit theorems of section 2 in an essential way. Finally, we note that our present analysis is restricted to 
the two-dimensional water wave problem, but the methods have the potential to extend to the fully three-dimensional water wave problem.

2. Limit theorems. We select the realizations of the bottom topography $\beta=\beta(x, \omega)$ from a stationary statistical ensemble $\omega \in \Omega$. That is, the probability space $(\Omega, \mathcal{M}, \mathrm{P})$ is equipped with a group of P-measure-preserving translations $\left\{\tau_{y}\right\}_{y \in \mathbb{R}}$ such that $\beta\left(x, \tau_{y} \omega\right)=\beta(x-y, \omega)$. We further assume that $\beta$ is $L^{\infty}(\mathbb{R})$ for a.e. realization $\omega \in \Omega$. We require that the measure be ergodic with respect to $\left\{\tau_{y}\right\}_{y \in \mathbb{R}}$ so that for P-a.e. realization $\omega$,

$$
\lim _{L \rightarrow \infty} \int_{0}^{L} F\left(\tau_{y} \omega\right) d y=\mathrm{E}(F)
$$

for every bounded measurable function $F: \Omega \rightarrow \mathbb{R}$. We further assume that there is an asymptotic decorrelation of typical realizations with respect to the probability measure $(\mathcal{M}, \mathrm{P})$, quantified by the strong mixing property $[8$, namely

$$
\left|\mathrm{P}\left(A \cap \tau_{y}(B)\right)-\mathrm{P}(A) \mathrm{P}(B)\right|<\alpha(y) \sqrt{\mathrm{P}(A) \mathrm{P}(B)},
$$

for sets $A \in \mathcal{M}_{\{y \leq 0\}}$ and $B \in \mathcal{M}_{\{y \geq 0\}}$, with the condition that the mixing rate satisfies

$$
\int_{0}^{\infty} \alpha^{1 / 2}(y) d y<+\infty \text {. }
$$

The covariance function of the process $\beta$ is defined by

$$
\rho_{\beta}(y)=\mathrm{E}\left(\beta(0, \omega) \beta\left(0, \tau_{y} \omega\right)\right),
$$

and its variance is

$$
\sigma_{\beta}^{2}=2 \int_{0}^{\infty} \rho_{\beta}(y) d y .
$$

We will actually need to analyse in detail the random process $\gamma(x, \omega)=\operatorname{sech}\left(D_{x}\right) \beta$. The process $\gamma$ inherits the properties of $\beta$; it is stationary, ergodic, of zero mean, and mixing. Its variance is expressed in terms of the variance of $\beta$ by the formula

$$
\sigma_{\gamma}^{2}=2 \int_{0}^{\infty} \operatorname{sech}^{2}\left(D_{y}\right) \rho_{\beta}(y) d y=\left.\operatorname{sech}^{2}(\xi) \hat{\rho}_{\beta}(\xi)\right|_{\xi=0}=\sigma_{\beta}^{2} .
$$

We note in particular that the process $\gamma$ is $C^{\infty}$ for a.e. realization $\omega \in \Omega$, due to the smoothing properties of the Fourier multiplier operator $\operatorname{sech}\left(D_{x}\right)$.

2.1. The invariance principle and functional central limit theorems. A key element in the derivation of the reduced system (1.1), (1.2) is the analysis of the influence of the rapid oscillations of the bottom, and in particular their possible homogenization and limiting effect on solutions. Donsker's invariance principle and the related functional central limit theorems provide the mathematical tools to address this question.

Theorem 2.1 (Billingsley [2]). Suppose that $\mu(x, \omega)$ is a stationary ergodic process which is mixing with a rate $\alpha$ that satisfies (2.3). Assume that $\mathrm{E}(\mu)=0$ and that $\sigma_{\mu} \neq 0$. Define

$$
Y_{\varepsilon}(\mu)(X)=\frac{\sqrt{\varepsilon}}{\sigma_{\mu}} \int_{0}^{\frac{X}{\varepsilon}} \mu(y) d y .
$$


As $\varepsilon$ tends to zero, $Y_{\varepsilon}$ converges in law to Brownian motion. That is, it satisfies

$$
Y_{\varepsilon}(\mu)(X) \Rightarrow B(X) \text {, }
$$

in the sense of weak convergence of probability measures on $C(\mathbb{R})$, where $B(X)=B_{\omega}(X)$ is a normalized Brownian motion.

A consequence of this result is the evaluation of integrals that are continuous functionals on path space evaluated on a family of processes whose limit is Brownian motion.

TheOREM 2.2. Let $\mu(x, \omega)$ be a stationary mixing ergodic process and $\varphi \in \mathcal{S}(\mathbb{R})$ be a test function. Then as $\varepsilon \rightarrow 0$,

$$
\int \mu(X / \varepsilon, \omega) \varphi(X) d X=\int\left(\mathrm{E}(\mu)+\sqrt{\varepsilon} \sigma_{\mu} \partial_{X} B_{\omega}(X)\right) \varphi(X) d X+o(\sqrt{\varepsilon}) .
$$

The equality is in the sense of convergence in law.

Proof. The first term is a consequence of the ergodic theorem and requires only that $\varphi \in L^{1}(\mathbb{R})$. The second term is a consequence of the Donsker invariance principle.

When less precise but uniform estimates are needed, the ergodic theorem implies the following.

CoROllary 2.3. Let $\mu(x, \omega)$ be a stationary mixing ergodic process of zero mean. Given $Y$ and $Y+t$ in a compact set and $\delta>0$, for a.e. realization $\omega \in \Omega$, there is an $\varepsilon_{0}=\varepsilon_{0}(\delta, \omega)$ such that for $\varepsilon<\varepsilon_{0}$,

$$
\left|\int_{0}^{t} \mu\left(\frac{Y+t^{\prime}}{\varepsilon}\right) d t^{\prime}\right| \leq \delta
$$

Proof. Write

$$
\int_{0}^{t} \mu\left(\frac{Y+t^{\prime}}{\varepsilon}\right) d t^{\prime}=\varepsilon \int_{0}^{\frac{Y+t}{\varepsilon}} \mu(s) d s-\varepsilon \int_{0}^{\frac{Y}{\varepsilon}} \mu(s) d s
$$

and suppose that $|Y|,|Y+t| \leq K$. Since $\mathrm{E}(\mu)=0$, for all $\delta>0$ there is $L_{0}=L_{0}(\delta, \omega)$ such that for all $L>L_{0}$,

$$
\frac{1}{L} \int_{0}^{L} \mu(s) d s<\frac{\delta}{2 K} .
$$

Hence, for $|Y|,|Y+t| \leq K$, for $\varepsilon<\varepsilon_{0}=\frac{\delta}{L_{0}|\mu|_{L} \infty}$, we have

$$
\left|\varepsilon \int_{0}^{\tilde{Y} / \varepsilon} \mu\left(t^{\prime}\right) d t^{\prime}\right|<\delta
$$

where $\tilde{Y}=Y$ or $Y+t$.

We will use these results to find the limiting asymptotic expressions for the solutions $(r, s)$ derived above, as well as in the estimate of trajectories and the consistency analysis There can be a difference in the function space setting. To distinguish between them, we use the following big $O$ and little $o$ notation.

(i) Pointwise convergence. A random variable $F_{\varepsilon, \omega}$ is said to converge pointwise at rate $\varepsilon^{m}$,

$$
\left.F_{\varepsilon, \omega}=O_{p}\left(\varepsilon^{m}\right) \quad \text { [respectively } o_{p}\left(\varepsilon^{m}\right)\right]
$$


as $\varepsilon \rightarrow 0$, when

$$
\varepsilon^{-m}\left|F_{\varepsilon, \omega}\right| \leq C(\omega)
$$

with the constant $C(\omega)$ a.e. finite [respectively, $<\delta(\omega)$ for arbitrary $\delta>0$ ].

(ii) Uniform convergence. We use the following notation to bound a random function $U(X, t, \varepsilon ; \omega)$ on compact sets of $(X, t) \in \mathbb{R}^{2}$ :

$$
U(X, t, \varepsilon ; \omega)=O_{\infty}(\varepsilon) \quad\left[\text { respectively } o_{\infty}(\varepsilon)\right]
$$

when as $\varepsilon \rightarrow 0$,

$$
\left|\varepsilon^{-m} U(X, t, \varepsilon ; \omega)\right|<C(\omega)
$$

uniformly over compact sets of $(X, t) \in \mathbb{R}^{2}$ [respectively, $<\delta(\omega)$ for arbitrary $\left.\delta>0\right]$. Corollary 2.3 gives an example of this form of limit.

(iii) Convergence in law on $C(\mathbb{R})$. A random function $U(x, t, \varepsilon ; \omega)$ is said to obey

$$
U(X, t, \varepsilon ; \omega)=O_{c}\left(\varepsilon^{m}\right) \quad\left[\text { respectively } o_{c}\left(\varepsilon^{m}\right)\right]
$$

if $\varepsilon^{-m} U(X, t, \varepsilon ; \omega)$ converge in law in $C(\mathbb{R})$. Theorem 2.1] in which $Y_{\varepsilon} \Rightarrow B_{\omega}$, is an example of this sort of convergence.

(iv) Convergence in law as a tempered distribution.

$$
U(X, t, \varepsilon ; \omega)=O_{d}\left(\varepsilon^{m}\right) \quad\left[\text { respectively } o_{d}\left(\varepsilon^{m}\right)\right]
$$

if for any test function $\varphi \in \mathcal{S}(\mathbb{R})$,

$$
F_{\varepsilon, \omega}:=\varepsilon^{-m} \int \varphi(X, t) U(X, t, \varepsilon ; \omega) d X d t \Rightarrow F_{0, \omega},
$$

for $F_{0, \omega}$ a random variable which is a.e. bounded. In particular, (iii) implies (iv). Theorem 2.1 gives an example of this form of convergence.

In the context of a random function $U(X, t, \varepsilon ; \omega)$, given a test function $\varphi \in \mathcal{S}\left(\mathbb{R}_{x} \times \mathbb{R}_{t}\right)$, if we have $U(X, t, \varepsilon ; \omega)=O_{d}\left(\varepsilon^{m}\right)$, this implies that

$$
\left|\varepsilon^{-m} \int \varphi(X, t) U(X, t, \varepsilon ; \omega) d X d t\right| \leq C(\omega)
$$

uniformly in $\varepsilon \rightarrow 0$, which in particular can be stated as

$$
F_{\varepsilon, \omega}=\int \varphi(X, t) U(X, t, \varepsilon ; \omega) d X d t=O_{p}\left(\varepsilon^{m}\right) \text {. }
$$

3. Euler equations and long wave scalings. The problem of water waves over a rapidly varying bottom can be viewed in the context of random homogenization theory and its corrections. We consider the Euler equations in Hamiltonian formulation, in which the topography has short scale variations, while the free surface $\eta$ and the boundary values of the velocity potential $\xi$ have a multiple scale description. An outline of the derivation of the effective equations is as follows. Let $u:=\partial_{x} \xi$ and consider $v=(\eta, u)$ in the appropriate phase space. Suppose that for small $\varepsilon>0$ the bottom topography varies rapidly, $\beta=\beta(X / \varepsilon)$, while the initial data and the subsequent solutions depend upon both the large scale variables $X$ and the small scale ones; namely, they appear in the form $v=v(X, t, \varepsilon)$. Solutions are then observed only with respect to the large 
scale variables. Being a Hamiltonian system, the equations of motion are given by the canonical equations

$$
\partial_{t} v=J \nabla_{v} H .
$$

Since we are principally interested in the large scale variations of the solutions, one actually observes

$$
\int \varphi(X) v(X, t, \varepsilon) d X
$$

for test functions $\varphi \in \mathcal{S}(\mathbb{R})$ which depend only on the large scale variables. Because of the structure of the Hamilton equations (3.1), the effective vector field is given by the principal asymptotic contributions of the quantities

$$
\int \varphi(X) J \nabla_{v} H(v(X, t, \varepsilon) d X .
$$

We assume that for the solutions we seek, this integral has an asymptotic description in the form

$$
\int \varphi(X) J \nabla_{v} H d X=\sum_{n=0}^{N} \varepsilon^{n} \int \varphi V^{(n)}(v) d X+o\left(\varepsilon^{N}\right) .
$$

Identifying the effective equations to order $O\left(\varepsilon^{N}\right)$ with the series, we have

$$
\partial_{t} v=\sum_{n=0}^{N} \varepsilon^{n} V^{(n)}(v) .
$$

These resulting equations need not be Hamiltonian, but they do describe the large scale asymptotic behavior of (3.1). We put this point of view into practice for the water wave problem in the following section.

In addition, we will find solutions which take the asymptotic form

$$
v=v^{(0)}(X, t)+\varepsilon v^{(1)}(X, t, \varepsilon ; \omega),
$$

where $v$ is uniformly bounded in an appropriate Sobolev space, independently of $\varepsilon, v^{(0)}$ is deterministic, and $v^{(1)}$ is random in that it depends on the particular realization at hand. This Ansatz will be verified a posteriori.

3.1. Hamiltonian formulation. The physical setting of the problem of water waves is to consider a potential flow in the fluid domain

$$
S(\eta, \beta)=\{-1+\beta(x)<y<\eta(x, t), x \in \mathbb{R}\},
$$

where the fluid depth has been normalized to $h=1$, and the free surface is given by $\{y=\eta(x, t)\}$. The equations of motion can be written in terms of a Hamiltonian

$$
\begin{aligned}
H & =\int_{\mathbb{R}} \int_{-1+\beta(x)}^{\eta(x)} \frac{1}{2}|\nabla \Phi(x, y)|^{2} d y d x+\int \frac{1}{2} \eta^{2}(x) d x \\
& =\int_{\mathbb{R}} \frac{1}{2} \xi(x) G(\beta, \eta) \xi(x) d x+\int \frac{1}{2} \eta^{2}(x) d x,
\end{aligned}
$$

where the acceleration of gravity $g$ is set to unity, and where $G(\beta, \eta)$ is the free surface Dirichlet-Neumann operator associated with the Laplace equation for the potential $\Phi$. 
The water wave evolution equations can be written in terms of the canonical variables $v(x)=(\eta(x), \xi(x)=\Phi(x, \eta(x)))$ in the form

$$
\partial_{t}\left(\begin{array}{l}
\eta \\
\xi
\end{array}\right)=\left(\begin{array}{cc}
0 & I \\
-I & 0
\end{array}\right) \nabla_{v} H
$$

where $\left(\begin{array}{cc}0 & I \\ -I & 0\end{array}\right)$ is presented in Darboux coordinates. We transform variables $(\eta, \xi)^{T} \mapsto$ $(\eta, u)^{T}$, for $u=\partial_{x} \xi$, under which the symplectic form is also transformed, so that

$$
J_{0} \rightarrow J_{1}=\left(\begin{array}{cc}
0 & -\partial_{x} \\
-\partial_{x} & 0
\end{array}\right)
$$

In the new variables,

$$
H(\eta, u)=\int_{\mathbb{R}} \frac{1}{2} u M(\eta, \beta) u d x+\int \frac{1}{2} \eta^{2} d x
$$

where $D M(\eta, \beta) D=G(\eta, \beta)$. We thus work with the evolution equations

$$
\begin{aligned}
& \partial_{t} \eta=-\partial_{x} \nabla_{u} H, \\
& \partial_{t} u=-\partial_{x} \nabla_{\eta} H .
\end{aligned}
$$

The detailed expansion of the Hamiltonian in terms of $\eta, \beta$ follows an analysis of the operator $M(\eta, \beta)[$ ], which can be written as

$$
\begin{aligned}
M(\eta, \beta) u & =D^{-1} \tanh (D) u+(\eta-\tanh (D) \eta \tanh (D)) u \\
& -\operatorname{sech}(D)(\beta+\beta D \tanh (D) \beta) \operatorname{sech}(D) u+T(\eta, \beta) u .
\end{aligned}
$$

The operator $T(\eta, \beta)$ is the result of a Taylor expansion, and for $u \in L^{2}(\mathbb{R})$, we have the estimate

$$
\|T(\eta, \beta) u\|_{L^{2}}=O\left(|\eta|_{C^{1}}^{2},|\beta|_{C^{1}}^{3},|\eta|_{C^{1}}|\beta|_{C^{1}}\right)\|u\|_{L^{2}} .
$$

3.2. Long wave scaling. Introduce the scaling regime for long wave, small amplitude evolution over a rapidly varying bottom; namely,

$$
\begin{array}{cl}
X=\varepsilon x, & \beta(x)=\varepsilon \tilde{\beta}(X / \varepsilon), \\
\eta(x, t)=\varepsilon^{2} \tilde{\eta}_{\varepsilon}(X, t), & u(x, t)=\varepsilon^{2} \tilde{u}_{\varepsilon}(X, t) .
\end{array}
$$

Transforming the Hamiltonian with this scaling, we are led to work with the system of equations based on

$$
\tilde{H}\left(\tilde{\eta}_{\varepsilon}, \tilde{u}_{\varepsilon} ; \varepsilon\right)=\frac{\varepsilon^{3}}{2} \int\left(\tilde{u}_{\varepsilon} M\left(\varepsilon^{2} \tilde{\eta}_{\varepsilon}, \varepsilon \tilde{\beta}_{\varepsilon}\right) \tilde{u}_{\varepsilon}+\tilde{\eta}_{\varepsilon}^{2}\right) d X .
$$

The expansion of $M$ and estimate (3.14) are used as a rationale for truncating the term $T$ in the Hamiltonian (although this does not constitute a rigorous justification). At this point, for notational simplicity, we drop the tilde:

$$
\begin{aligned}
& H\left(\eta_{\varepsilon}, u_{\varepsilon} ; \varepsilon\right)=\frac{\varepsilon^{3}}{2} \int\left(u_{\varepsilon}\left(\varepsilon D_{X}\right)^{-1} \tanh \left(\varepsilon D_{X}\right) u_{\varepsilon}+\eta_{\varepsilon}^{2}\right. \\
& \quad+\varepsilon^{2} u_{\varepsilon}\left(\eta_{\varepsilon}-\tanh \left(\varepsilon D_{X}\right) \eta_{\varepsilon} \tanh \left(\varepsilon D_{X}\right)\right) u_{\varepsilon} \\
& \left.\quad-u_{\varepsilon} \operatorname{sech}\left(\varepsilon D_{X}\right)\left[\varepsilon \beta+\varepsilon^{2} \beta\left(\varepsilon D_{X}\right) \tanh \left(\varepsilon D_{X}\right) \beta\right] \operatorname{sech}\left(\varepsilon D_{X}\right) u_{\varepsilon}\right) d X .
\end{aligned}
$$


The symplectic form is transformed to

$$
J=\varepsilon^{-3}\left(\begin{array}{cc}
0 & -\partial_{X} \\
-\partial_{X} & 0
\end{array}\right) .
$$

From this expression, we derive the principal asymptotic contributions to the resulting Hamiltonian vector field using the criterion of (3.4) (with $N=2$ );

$$
\partial_{t} \eta_{\varepsilon}=V_{1}\left(\eta_{\varepsilon}, u_{\varepsilon} ; \varepsilon\right), \quad \partial_{t} u_{\varepsilon}=V_{2}\left(\eta_{\varepsilon}, u_{\varepsilon} ; \varepsilon\right)
$$

where for test functions $\varphi \in \mathcal{S}(\mathbb{R})$, we have

$$
\begin{aligned}
& \int-\partial_{X} \nabla_{u} H \varphi d X=\int V_{1} \varphi d X+o_{p}\left(\varepsilon^{2}\right), \\
& \int-\partial_{X} \nabla_{\eta} H \varphi d X=\int V_{2} \varphi d X+o_{p}\left(\varepsilon^{2}\right) .
\end{aligned}
$$

To determine the components $V_{1}$ and $V_{2}$, one must perform an expansion of multiple scales operators. The following two lemmas of [3] are useful for this.

Lemma 3.1. Let $m(k)$ be a Fourier multiplier which is a classical symbol of order $r$ and (for convenience) consider $J \geq r$. For fixed $f(X) \in H^{J}\left(\mathbb{R}^{d}\right)$, then

$$
\left\|\left(m\left(\varepsilon D_{X}\right)-\sum_{\alpha:|\alpha|=0}^{J} \frac{\varepsilon^{\alpha}}{\alpha !} m^{(\alpha)}(0) D_{X}^{\alpha}\right) f\right\|_{L^{2}}:=\left\|R_{\varepsilon}^{(J)} f\right\|_{L^{2}} \leq o\left(\varepsilon^{J}\right)\left\|D_{X}^{J} f\right\|_{L^{2}} .
$$

Lemma 3.2. Again let $m(k)$ be a classical symbol of order $r$, and take $J \geq r$. Assume that $m(k)$ satisfies the condition that

$$
\sum_{\alpha:|\alpha|=J+1} \int\left|\partial_{k}^{\alpha} m(k)\right| d k<+\infty
$$

(this is automatically satisfied if $J \geq r+d)$. Fix $f(X) \in H^{J}\left(\mathbb{R}^{d}\right)$, and suppose that $\beta(x) \in C^{J}$. Then

$$
\begin{aligned}
& \left\|\left(m\left(\varepsilon D_{X}\right)(\beta f)-\sum_{\alpha:|\alpha|=0}^{J} \frac{\varepsilon^{\alpha}}{\alpha !}\left(m^{(\alpha)}\left(D_{x}\right) \beta\right) D_{X}^{\alpha}\right) f\right\|_{L^{2}}:=\left\|R_{\varepsilon}^{(J)}(\beta) f\right\|_{L^{2}} \\
& \quad \leq o_{\varepsilon}(1)\left(\varepsilon^{J}|\beta|_{C^{0}}\left\|D_{X}^{J} f\right\|_{L^{2}}+|\beta|_{C^{J}}\|f\|_{L^{2}}\right) .
\end{aligned}
$$

The standard Fourier multipliers associated with the Dirichlet-Neumann operator in the fluid domain will satisfy the extra condition (3.21). The cases at hand are typically

$$
\begin{aligned}
& m_{1}(k)=\operatorname{sech}(h k), \quad r=0, \quad J \geq 0, \\
& m_{2}(k)=k \tanh (h k), \quad r=1, \quad J \geq 1 .
\end{aligned}
$$

3.3. Effective system of equations. We assume that for fixed $t,\left(u_{\varepsilon}, \eta_{\varepsilon}\right)$ are bounded in $H^{1}$, uniformly in $\varepsilon$, a.e. in $\omega$, and have the form

$$
\begin{aligned}
& u_{\varepsilon}=u_{0}(X, t)+\varepsilon u_{1}(X, t ; \varepsilon), \\
& \eta_{\varepsilon}=\eta_{0}(X, t)+\varepsilon \eta_{1}(X, t ; \varepsilon) .
\end{aligned}
$$

The principal parts $\left(u_{0}, \eta_{0}\right)$ are deterministic functions of the large scale variable $X$, and $\left(u_{1}, \eta_{1}\right)$ are the first corrections. Giving initial data for the equation (1.3) in $H^{1}(\mathbb{R})$ 
$\left(\right.$ or in $\mathcal{S}$ ) the quantities $\left(\eta_{0}, u_{0}\right)$ inherit this property. The correction terms $\left(u_{1}, \eta_{1}\right)$ are asymptotically more irregular, as they have properties of randomness. These Ansätze have to be verified, which will be done after deriving formulas for the solution in section 4 .

Proposition 3.3. Under the above Ansatz, the vector field $\left(V_{1}, V_{2}\right)$ defined in (3.19) and $(3.20)$ is

$$
\begin{aligned}
& V_{1}=-\partial_{X}\left(h_{\varepsilon}(X) u_{\varepsilon}+\varepsilon^{2} \eta_{\varepsilon} u_{\varepsilon}+\frac{\varepsilon^{2}}{3} \partial_{X}^{2} u_{\varepsilon}\right), \\
& V_{2}=-\partial_{X}\left(\eta_{\varepsilon}+\frac{\varepsilon^{2}}{2} u_{\varepsilon}^{2}\right),
\end{aligned}
$$

where the corrected depth $h_{\varepsilon}$ is given as

$$
h_{\varepsilon}(X)=1-\varepsilon \gamma(X / \varepsilon)-\varepsilon^{2} a_{\beta},
$$

$\gamma=\operatorname{sech}\left(D_{x}\right) \beta$ and $a_{\beta}=\mathrm{E}\left(\beta D_{x} \tanh \left(D_{x}\right) \beta\right)$.

Proof. The goal is to compute the l.h.s. of (3.19) and (3.20) up to order $O\left(\varepsilon^{2}\right)$ :

$$
\begin{aligned}
\int- & \partial_{X} \nabla_{u} H \varphi d X=\int \varphi_{X}\left(\left(\varepsilon D_{X}\right)^{-1} \tanh \left(\varepsilon D_{X}\right) u_{\varepsilon}\right. \\
& +\varepsilon^{2}\left(\eta_{\varepsilon}-\tanh \left(\varepsilon D_{X}\right) \eta_{\varepsilon} \tanh \left(\varepsilon D_{X}\right)\right) u_{\varepsilon} \\
& \left.-\operatorname{sech}\left(\varepsilon D_{X}\right)\left(\varepsilon \beta+\varepsilon^{2} \beta\left(\varepsilon D_{X}\right) \tanh \left(\varepsilon D_{X}\right) \beta\right) \operatorname{sech}\left(\varepsilon D_{X}\right) u_{\varepsilon}\right) d X+o_{p}\left(\varepsilon^{2}\right),
\end{aligned}
$$

and

$$
\int-\partial_{X} \nabla_{\eta} H \varphi d X=\int \varphi_{X}\left(\eta_{\varepsilon}+\frac{\varepsilon^{2}}{2}\left[u_{\varepsilon}-\left(\tanh \left(\varepsilon D_{X}\right) u_{\varepsilon}\right)^{2}\right]\right) d X+o_{p}\left(\varepsilon^{2}\right) \cdot(3
$$

We calculate the various terms in the l.h.s. of (3.30) that we denote $I_{j}(j=1, \ldots, 4)$ :

$$
I_{1}=\int \varphi_{X}\left(\varepsilon D_{X}\right)^{-1} \tanh \left(\varepsilon D_{X}\right) u_{\varepsilon} d X=\int\left(\left(\varepsilon D_{X}\right)^{-1} \tanh \left(\varepsilon D_{X}\right) \varphi_{X}\right) u_{\varepsilon} d X .
$$

We apply Lemma 3.1 to evaluate $\left(\varepsilon D_{X}\right)^{-1} \tanh \left(\varepsilon D_{X}\right) \varphi_{X}$, to the effect that

$$
I_{1}=\int u_{\varepsilon}\left(1-\frac{\varepsilon^{2}}{3} D_{X}^{2}\right) \varphi_{X} d X+O_{p}\left(\varepsilon^{4}\right) .
$$

The contribution of $I_{1}$ to $V_{1}$ is $-\partial_{X}\left(1+\frac{\varepsilon^{2}}{3} \partial_{X}^{2}\right) u_{\varepsilon}$. We now turn to $I_{2}$ defined as

$$
I_{2}=\varepsilon^{2} \int \varphi_{X}\left(\eta_{\varepsilon}-\tanh \left(\varepsilon D_{X}\right) \eta_{\varepsilon} \tanh \left(\varepsilon D_{X}\right)\right) u_{\varepsilon} d X .
$$

Since $\left|\tanh \left(\varepsilon D_{X}\right) u_{\varepsilon}\right|_{L^{2}} \leq\left|u_{\varepsilon}\right|_{L^{2}}$, we immediately deduce that

$$
I_{2}=\varepsilon^{2} \int \varphi_{X} \eta_{\varepsilon} u_{\varepsilon} d X+O_{p}\left(\varepsilon^{3}\right)
$$

and its contribution to $V_{1}$ is $-\varepsilon^{2} \partial_{X}\left(\eta_{\varepsilon} u_{\varepsilon}\right)$. The third term $I_{3}$ is

$$
I_{3}=-\varepsilon \int \varphi_{X} \operatorname{sech}\left(\varepsilon D_{X}\right) \beta \operatorname{sech}\left(\varepsilon D_{X}\right) u_{\varepsilon} d X=-\varepsilon \int\left(\operatorname{sech}\left(\varepsilon D_{X}\right) \varphi_{X}\right) \beta \operatorname{sech}\left(\varepsilon D_{X}\right) u_{\varepsilon} d X \text {. }
$$


From Lemma 3.1,

$$
I_{3}=-\varepsilon \int \varphi_{X} \beta \operatorname{sech}\left(\varepsilon D_{X}\right) u_{\varepsilon} d X+O\left(\varepsilon^{3}\right)=-\varepsilon \int u_{\varepsilon} \operatorname{sech}\left(\varepsilon D_{X}\right)\left(\varphi_{X} \beta\right) d X+O_{p}\left(\varepsilon^{3}\right) .
$$

We now use Lemma 3.2 to find

$$
I_{3}=-\varepsilon \int u_{\varepsilon}\left[\left(\operatorname{sech}\left(D_{x}\right) \beta\right) \varphi_{X}+\varepsilon\left(\operatorname{sech}\left(D_{x}\right) \tanh \left(D_{x}\right) \beta\right) \partial_{X}^{2} \varphi\right] d X+O_{p}\left(\varepsilon^{3}\right) .
$$

The contribution of the first term of $I_{3}$ to $V_{1}$ is $-\varepsilon \partial_{X}\left(-\left(\operatorname{sech}\left(D_{x}\right) \beta\right) u_{\varepsilon}\right)$. To evaluate the second term of $I_{3}$, we use the Ansatz (3.25) and apply Corollary 2.3.

$$
\begin{aligned}
& \int\left(\operatorname{sech}\left(D_{x}\right) \tanh \left(D_{x}\right) \beta\right) \partial_{X}^{2} \varphi u_{\varepsilon} d X=\int\left(\operatorname{sech}\left(D_{x}\right) \tanh \left(D_{x}\right) \beta\right) \partial_{X}^{2} \varphi u_{0} d X+O_{p}(\varepsilon) \\
& =\mathrm{E}\left(\operatorname{sech}\left(D_{x}\right) \tanh \left(D_{x}\right) \beta\right) \int \partial_{X}^{2} \varphi u_{0} d X+o_{p}(1) .
\end{aligned}
$$

Since $\mathrm{E}\left(\operatorname{sech}\left(D_{x}\right) \tanh \left(D_{x}\right) \beta\right)=0$, the second term of $I_{3}$ thus has a contribution to $V_{1}$ at order $o_{p}\left(\varepsilon^{2}\right)$. Finally we examine

$$
I_{4}=-\varepsilon^{2} \int \varphi_{X} \operatorname{sech}\left(\varepsilon D_{X}\right) \beta\left(\varepsilon D_{X}\right) \tanh \left(\varepsilon D_{X}\right) \beta \operatorname{sech}\left(\varepsilon D_{X}\right) u_{\varepsilon} d X
$$

and proceed similarly. We find that

$$
\begin{aligned}
I_{4} & =-\varepsilon^{2} \int \varphi_{X} \beta\left(\varepsilon D_{X}\right) \tanh \left(\varepsilon D_{X}\right) \beta \operatorname{sech}\left(\varepsilon D_{X}\right) u_{\varepsilon} d X+O_{p}\left(\varepsilon^{4}\right) \\
& =-\varepsilon^{2} \int\left(\varphi_{X} \beta\left(\varepsilon D_{X}\right) \tanh \left(\varepsilon D_{X}\right) \beta\right) \operatorname{sech}\left(\varepsilon D_{X}\right) u_{0} d X+O_{p}\left(\varepsilon^{3}\right) \\
& =-\varepsilon^{2} \int\left(\varphi_{X} \beta\left(\varepsilon D_{X}\right) \tanh \left(\varepsilon D_{X}\right) \beta\right) u_{0} d X+O_{p}\left(\varepsilon^{3}\right) \\
& =-\varepsilon^{2} \int\left(\beta D_{x} \tanh \left(D_{x}\right) \beta\right) \varphi_{X} u_{0} d X+O_{p}\left(\varepsilon^{3}\right) .
\end{aligned}
$$

Denoting $a_{\beta}=\mathrm{E}\left(\beta D_{x} \tanh \left(D_{x}\right) \beta\right)$ the term $I_{4}$ reduces to

$$
I_{4}=-\varepsilon^{2} a_{\beta} \int \varphi_{X} u_{\varepsilon}+O_{p}\left(\varepsilon^{3}\right),
$$

and its contribution to $V_{1}$ is $-\partial_{X}\left(-\varepsilon^{2} a_{\beta} u_{\varepsilon}\right)$. This concludes the derivation of (3.27) in Proposition 3.3 .

We now turn to the evaluation of (3.31). Using the Ansatz (3.25), we have that

$$
\varepsilon^{2} \int \varphi_{X}\left(\tanh \left(\varepsilon D_{X}\right) u_{\varepsilon}\right)^{2} d X=\varepsilon^{2} \int \varphi_{X}\left(\tanh \left(\varepsilon D_{X}\right) u_{0}\right)^{2}+O_{p}\left(\varepsilon^{3}\right) .
$$

The resulting formula for $V_{2}$ is straightforward.

3.4. The $K d V$ regime. To describe the $\mathrm{KdV}$ regime, an additional change of variables is performed in the form

$$
\eta_{\varepsilon}=\frac{1}{2 k_{\varepsilon}}\left(r_{\varepsilon}+\varepsilon^{3 / 2} s_{\varepsilon}\right) ; u_{\varepsilon}=k_{\varepsilon}\left(r_{\varepsilon}-\varepsilon^{3 / 2} s_{\varepsilon}\right)
$$

with $k_{\varepsilon}=\left(4 h_{\varepsilon}\right)^{-1 / 4}$ and the corrected depth $h_{\varepsilon}$ is given in (3.29). The functions $r_{\varepsilon}$ and $s_{\varepsilon}$ can be seen as the components of the solution traveling to the right and left, respectively. The presence of the scaling factor $\varepsilon^{3 / 2}$ in front of $s_{\varepsilon}$ is to put forward the 
assumption that $r_{\varepsilon}$ is the main component of the solution while $s_{\varepsilon}$ represents the 'small' scattered component. The resulting system for $\left(r_{\varepsilon}, s_{\varepsilon}\right)$ is (after dropping the subscript $\varepsilon$ )

$$
\begin{gathered}
\partial_{t} r=-\partial_{X}\left[\sqrt{g h_{\varepsilon}} r+\frac{\varepsilon^{2} h^{3}}{3} k_{\varepsilon} \partial_{X}^{2}\left(k_{\varepsilon} r-\varepsilon^{3 / 2} k_{\varepsilon} s\right)+\frac{\varepsilon^{2}}{4} k_{\varepsilon}\left(3 r^{2}-2 \varepsilon^{3 / 2} r s-\varepsilon^{3} s^{2}\right)\right] \\
+\frac{1}{4} \frac{\partial_{X} h_{\varepsilon}}{h_{\varepsilon}}\left[\varepsilon^{3 / 2} \sqrt{g h_{\varepsilon}} s-\frac{\varepsilon^{2} h^{3}}{3} k_{\varepsilon} \partial_{X}^{2}\left(k_{\varepsilon} r-\varepsilon^{3 / 2} k_{\varepsilon} s\right)+\frac{\varepsilon^{2}}{4} k_{\varepsilon}\left(-r^{2}-2 \varepsilon^{3 / 2} r s+3 \varepsilon^{3} s^{2}\right)\right] \\
\partial_{t} s=\partial_{X}\left[\sqrt{g h_{\varepsilon}} s-\frac{\varepsilon^{2} h^{3}}{3} k_{\varepsilon} \partial_{X}^{2}\left(k_{\varepsilon} \varepsilon^{-3 / 2} r-k_{\varepsilon} s\right)+\frac{\varepsilon^{2}}{4} k_{\varepsilon}\left(-\varepsilon^{-3 / 2} r^{2}-2 r s+3 \varepsilon^{3 / 2} s^{2}\right)\right] \\
-\frac{1}{4} \frac{\partial_{X} h_{\varepsilon}}{h_{\varepsilon}}\left[\varepsilon^{-3 / 2} \sqrt{g h_{\varepsilon}} r+\frac{\varepsilon^{1 / 2} h^{3}}{3} k_{\varepsilon} \partial_{X}^{2}\left(k_{\varepsilon} r-k_{\varepsilon} \varepsilon^{3 / 2} s\right)+\frac{\varepsilon^{1 / 2}}{4} k_{\varepsilon}\left(3 r^{2}-2 \varepsilon^{3 / 2} r s-\varepsilon^{3} s^{2}\right)\right] .
\end{gathered}
$$

There are clearly more terms in (3.45), (3.46) than in the model system (1.1), (1.2). To make the selection of the dominant terms that are retained, one must understand their asymptotic contribution as per the criterion expressed in (3.4), when evaluated on solutions of (1.1), (1.2). This consistency analysis is carried out in detail in [3, where it is shown that only the two parameters $a, b$ are needed to express the difference between (3.45), (3.46) and (1.1), (1.2), up to errors of order $o\left(\varepsilon^{2}\right)$. We refer to 3 for the details of this procedure.

4. Dispersive system with oscillating coefficients. The link between equations (1.1) and (1.3) involves a transformation to random characteristic coordinates, defined using the corrected wavespeed $c_{\varepsilon}(X, \omega)$. The characteristics, and their asymptotic behavior as $\varepsilon$ tends to zero, is the first topic of this section. We secondly address the ensuing change of variables for the equation (1.3), for which it is important that we consider the limit of solutions in the sense of tempered distributions.

4.1. Characteristic coordinates. When solving the system (1.1), (1.2), we are led to consider trajectories of the flow of a random equation

$$
\frac{d X}{d t}=c_{\varepsilon}(X, \omega):=\left(1-\varepsilon \mu\left(\frac{X}{\varepsilon}\right)-\varepsilon^{2} a\right), \quad X(0 ; Y)=Y .
$$

The realizations of the random process $\mu$ are taken from a statistical ensemble $\omega \in \Omega$, and we assume that the process is stationary, ergodic, with zero mean and asymptotically mixing. We also need that $\mu(x, \omega) \in C^{3}$ for P-a.e. realization $\omega$. (This assumption will be satisfied when we apply these results to $\mu=\frac{1}{2} \operatorname{sech}\left(D_{x}\right) \beta$.) Thus the characteristic vector field $c_{\varepsilon}(X, \omega)$ is $C^{1}$, and for a given realization $\omega$, uniformly in $\varepsilon$. The parameter $a$ is constant.

Theorem 4.1. The expansion of the trajectories in $\varepsilon$ are given by

$$
X(t ; Y, \varepsilon)=Y+t-\varepsilon^{3 / 2}\left(\varepsilon^{-1 / 2} \int_{0}^{t} \mu\left(\frac{Y+t^{\prime}}{\varepsilon}\right) d t^{\prime}\right)-\varepsilon^{2}\left(a+\mathrm{E}\left(\mu^{2}\right)\right) t+o_{\infty}\left(\varepsilon^{2}\right) .
$$

Inverting this relationship,

$$
Y(t ; X, \varepsilon)=X-t+\varepsilon^{3 / 2}\left(\varepsilon^{-1 / 2} \int_{0}^{t} \mu\left(\frac{X+\left(t^{\prime}-t\right)}{\varepsilon}\right) d t^{\prime}\right)+\varepsilon^{2}\left(a+\mathrm{E}\left(\mu^{2}\right)\right) t+o_{\infty}\left(\varepsilon^{2}\right) .
$$


Proof. Since $c_{\varepsilon}$ is a $C^{1}$ function of $X$ over $\varepsilon \in\left(0, \varepsilon_{0}\right)$, the trajectories $X_{\varepsilon}(t ; Y)$ exist for all time $t$, the flow $\varphi_{t}^{\varepsilon}(Y)=X_{\varepsilon}(t ; Y)$ is $C^{1}$ and the mapping $\left\{(t, Y) \rightarrow\left(t, X_{\varepsilon}\right), 0<\varepsilon<\varepsilon_{0}\right\}$ is compact in the weak topology. To describe the asymptotic behavior of the trajectories, let $X_{\varepsilon}(t)=X_{0}+\varepsilon Z$, where $X_{0}(t)=Y+t$ and

$$
\begin{aligned}
& \dot{Z}(t)=-\mu\left(\frac{Y+t}{\varepsilon}+Z(t)\right)-\varepsilon a \\
& =-\mu\left(\frac{Y+t}{\varepsilon}\right)-Z \mu^{\prime}\left(\frac{Y+t}{\varepsilon}\right)-Z^{2} \int_{0}^{1}(1-s) \mu^{\prime \prime}\left(\frac{Y+t}{\varepsilon}+s Z(t)\right) d s-\varepsilon a .
\end{aligned}
$$

Write $Z=\varepsilon^{1 / 2} Z_{1}+\varepsilon Z_{2}$. Then (4.4) is equivalent to the system

$$
\begin{aligned}
& \dot{Z}_{1}(t)=-\varepsilon^{-1 / 2} \mu\left(\frac{Y+t}{\varepsilon}\right)-\mu^{\prime}\left(\frac{Y+t}{\varepsilon}\right) Z_{1}, \\
& \dot{Z}_{2}(t)=-\mu^{\prime}\left(\frac{Y+t}{\varepsilon}\right) Z_{2} \\
& \quad-\left(Z_{1}+\varepsilon^{1 / 2} Z_{2}\right)^{2} \int_{0}^{1}(1-s) \mu^{\prime \prime}\left(\frac{Y+t}{\varepsilon}+s\left(\varepsilon^{1 / 2} Z_{1}+\varepsilon Z_{2}\right)\right) d s-a .
\end{aligned}
$$

Define $W_{j}=p_{\varepsilon}(t) Z_{j}(t)(j=1,2)$ with the integrating factor

$$
p_{\varepsilon}(t)=C e^{\int_{0}^{t} \mu^{\prime}\left(\frac{Y+t^{\prime}}{\varepsilon}\right) d t^{\prime}}=C e^{\varepsilon \mu\left(\frac{Y+t}{\varepsilon}\right)} e^{-\varepsilon \mu\left(\frac{Y}{\varepsilon}\right)} .
$$

Setting $C=C(Y, \varepsilon, \omega)=e^{\varepsilon \mu\left(\frac{Y}{\varepsilon}\right)}$ gives a simpler form for $W_{j}$; indeed,

$$
W_{j}=Z_{j} e^{\varepsilon \mu\left(\frac{Y+t}{\varepsilon}\right)}(j=1,2) .
$$

In terms of $W_{1}, W_{2}$, the characteristic equations (4.6) become

$$
\begin{aligned}
\dot{W}_{1}(t)= & -\varepsilon^{-1 / 2} \mu\left(\frac{Y+t}{\varepsilon}\right) p_{\varepsilon}(t), \\
\dot{W}_{2}(t)= & -\left(W_{1} p_{\varepsilon}^{-1}+\varepsilon^{1 / 2} p_{\varepsilon}^{-1} W_{2}\right)^{2} \int_{0}^{1}(1-s) \mu^{\prime \prime}\left(\frac{Y+t}{\varepsilon}+s\left(\varepsilon^{1 / 2} W_{1} p_{\varepsilon}^{-1}+\varepsilon p_{\varepsilon}^{-1} W_{2}\right)\right) d s \\
& -a p_{\varepsilon}^{-1} .
\end{aligned}
$$

The first equation is solved by quadrature, namely

$$
\begin{aligned}
& W_{1}(t ; Y, \varepsilon)=-\varepsilon^{-1 / 2} \int_{0}^{t} \mu\left(\frac{Y+t^{\prime}}{\varepsilon}\right) p_{\varepsilon}\left(t^{\prime}\right) d t^{\prime} \\
& =-\varepsilon^{-1 / 2} \int_{0}^{t} \mu\left(\frac{Y+t^{\prime}}{\varepsilon}\right) d t^{\prime}-\varepsilon^{1 / 2} \int_{0}^{t} \mu^{2}\left(\frac{Y+t^{\prime}}{\varepsilon}\right) d t^{\prime}-\varepsilon^{1 / 2} \int_{0}^{t} Q(\mu, \varepsilon) d t^{\prime}
\end{aligned}
$$

with

$$
Q=\mu\left(\frac{Y+t}{\varepsilon}\right)\left(e^{\varepsilon \mu\left(\frac{Y+t}{\varepsilon}\right)}-1-\varepsilon \mu\left(\frac{Y+t}{\varepsilon}\right)\right) .
$$

The asymptotic behavior of $W_{1}$ is therefore

$$
W_{1}(t ; Y, \varepsilon)=-\varepsilon^{-1 / 2} \int_{0}^{t} \mu\left(\frac{Y+t^{\prime}}{\varepsilon}\right) d t^{\prime}-\varepsilon^{1 / 2} \mathrm{E}\left(\mu^{2}\right) t+R_{\varepsilon},
$$

where $R_{\varepsilon}$ is given by

$$
R_{\varepsilon}=-\varepsilon^{1 / 2} \int_{0}^{t}\left(\mu^{2}\left(\frac{Y+t}{\varepsilon}\right)-\mathrm{E}\left(\mu^{2}\right)\right) d t^{\prime}-\varepsilon^{1 / 2} \int_{0}^{t} Q(\mu, \varepsilon) d t^{\prime} .
$$


The quantity $|Q(\mu)|_{L^{\infty}} \leq\left|\varepsilon^{2} \mu^{2} e^{\mu}\right|_{L^{\infty}}$; thus it is $O_{\infty}\left(\varepsilon^{2}\right)$ since $|\mu|_{C^{1}}$ is bounded for a.e. $\omega$. The first term on the other hand is described by

$$
\varepsilon\left(\varepsilon^{-1 / 2} \int_{0}^{t}\left[\mu^{2}\left(\frac{Y+t}{\varepsilon}\right)-\mathrm{E}\left(\mu^{2}\right)\right] d t^{\prime}\right)
$$

for which $\left(\varepsilon^{-1 / 2} \int_{0}^{t}\left[\mu^{2}\left(\frac{Y+t}{\varepsilon}\right)-\mathrm{E}\left(\mu^{2}\right)\right] d t^{\prime}\right)$ converges in law on the space $C[0, T]$ to Brownian motion. Hence it is $O_{c}(\varepsilon)$ in the asymptotic sense described in section 2 ,

When applying Corollary 2.3 to $\mu^{2}-\mathrm{E}\left(\mu^{2}\right)$, we get

$$
\varepsilon^{1 / 2} \int_{0}^{t}\left[\mu^{2}\left(\frac{Y+t}{\varepsilon}\right)-\mathrm{E}\left(\mu^{2}\right)\right] d t^{\prime}=o_{\infty}\left(\varepsilon^{1 / 2}\right) .
$$

As a consequence, we also have the asymptotic behavior of $Z_{1}$ in the form

$$
\begin{aligned}
& Z_{1}(t)=W_{1}(t) p_{\varepsilon}^{-1}=\left[-\varepsilon^{-1 / 2} \int_{0}^{t} \mu\left(\frac{Y+t^{\prime}}{\varepsilon}\right) d t^{\prime}+\varepsilon^{1 / 2} \mathrm{E}\left(\mu^{2}\right) t\right] e^{-\varepsilon \mu\left(\frac{Y+t}{\varepsilon}\right)}+O_{\infty}(\varepsilon) \\
& =-\varepsilon^{-1 / 2} \int_{0}^{t} \mu\left(\frac{Y+t^{\prime}}{\varepsilon}\right) d t^{\prime}+\varepsilon^{1 / 2} \mu\left(\frac{Y+t}{\varepsilon}\right) \int_{0}^{t} \mu\left(\frac{Y+t^{\prime}}{\varepsilon}\right) d t^{\prime}-\varepsilon^{1 / 2} \mathrm{E}\left(\mu^{2}\right) t \\
& +o_{\infty}\left(\varepsilon^{1 / 2}\right) .
\end{aligned}
$$

The term of (4.16) of the form $\varepsilon^{1 / 2} \mu\left(\frac{Y+t}{\varepsilon}\right) \int_{0}^{t} \mu\left(\frac{Y+t^{\prime}}{\varepsilon}\right) d t^{\prime}$ is bounded by $o_{\infty}\left(\varepsilon^{1 / 2}\right)$ since $|\mu|_{L^{\infty}}<C$, and Corollary 2.3 bounds the remaining integral by ergodicity. Consequently,

$$
Z_{1}(t)=-\varepsilon^{-1 / 2} \int_{0}^{t} \mu\left(\frac{Y+t^{\prime}}{\varepsilon}\right) d t^{\prime}-\varepsilon^{1 / 2} \mathrm{E}\left(\mu^{2}\right)+o_{\infty}\left(\varepsilon^{1 / 2}\right) .
$$

By the invariance principle (Section 2), the asymptotic behavior of $Z_{1}(t)$ is described by Brownian motion, indeed in the sense of convergence in law:

$$
Z_{1}(t) \sim-\sigma_{\mu} B_{\omega(Y)}(t)-\varepsilon^{1 / 2} \mathrm{E}\left(\mu^{2}\right) .
$$

Now turn to the expression for the error $W_{2}(t ; Y, \varepsilon)$. The purpose of the analysis is to give $\varepsilon$-independent bounds for $W_{2}$ to complete the verification of the asymptotic behavior of the series for $X$. The function $W_{2}$ is given by the integral equation

$$
\begin{aligned}
& W_{2}(t)=-\int_{0}^{t}\left(Z_{1}+\varepsilon^{1 / 2} p_{\varepsilon}^{-1} W_{2}\right)^{2} \int_{0}^{1}(1-s) \mu^{\prime \prime}\left(\frac{Y+t^{\prime}}{\varepsilon}+s\left(\varepsilon^{1 / 2} Z_{1}+\varepsilon p_{\varepsilon}^{-1} W_{2}\right)\right) d s d t^{\prime} \\
& -a \int_{0}^{t} p_{\varepsilon}^{-1}\left(t^{\prime}\right) d t^{\prime}=F\left(W_{2}\right)(t)
\end{aligned}
$$

where $F(W)$ is considered as a mapping on functions $W(t)$. For a contraction argument,

$$
F\left(W_{2}\right)-F\left(\tilde{W}_{2}\right)=I_{1}+I_{2},
$$


where

$$
\begin{aligned}
I_{1}= & -\int_{0}^{t}\left(Z_{1}+\varepsilon^{1 / 2} p_{\varepsilon}^{-1} W_{2}\right)^{2} \int_{0}^{1}(1-s)\left[\mu^{\prime \prime}\left(\frac{Y+t^{\prime}}{\varepsilon}+s\left(\varepsilon^{1 / 2} Z_{1}+\varepsilon p_{\varepsilon}^{-1} W_{2}\right)\right)\right. \\
& \left.-\mu^{\prime \prime}\left(\frac{Y+t^{\prime}}{\varepsilon}+s\left(\varepsilon^{1 / 2} Z_{1}+\varepsilon p_{\varepsilon}^{-1} \tilde{W}_{2}\right)\right)\right] d s d t^{\prime} \\
I_{2}= & -\int_{0}^{t}\left[\left(Z_{1}+\varepsilon^{1 / 2} p_{\varepsilon}^{-1} W_{2}\right)^{2}-\left(Z_{1}+\varepsilon^{1 / 2} p_{\varepsilon}^{-1} \tilde{W}_{2}\right)^{2}\right] \\
& \times \int_{0}^{1}(1-s) \mu^{\prime \prime}\left(\frac{Y+t^{\prime}}{\varepsilon}+s\left(\varepsilon^{1 / 2} Z_{1}+\varepsilon p_{\varepsilon}^{-1} \tilde{W}_{2}\right)\right) d s d t^{\prime}
\end{aligned}
$$

Suppose that $W_{2}, \tilde{W}_{2} \in B_{R}(0) \subseteq C^{0}\left[0, T_{0}\right]$. We will obtain a Lipschitz estimate for each of these two terms.

For the term $I_{1}$, consider the two factors separately. The first factor is bounded by the result of Corollary 2.3, indeed for $|Y|,|Y+t|<K$, and $\varepsilon<\varepsilon_{0}=\varepsilon_{0}(\delta, \omega)$. Then

$$
\left|Z_{1}+\varepsilon^{1 / 2} p_{\varepsilon}^{-1} W_{2}\right|^{2} \leq\left(\varepsilon^{-1 / 2} \delta+\varepsilon^{1 / 2} R\right)^{2} .
$$

The second factor is responsible for the contraction

$$
\begin{aligned}
& \left.\varepsilon\left|\int_{0}^{1}(1-s)\right| \mu^{\prime \prime}\right|_{\operatorname{Lip}}\left|W_{2}\left(t^{\prime}\right)-\tilde{W}_{2}\left(t^{\prime}\right)\right| p_{\varepsilon}^{-1} d s \\
& \quad \leq 2 \varepsilon\left|\mu^{\prime \prime}\right|_{\operatorname{Lip}}\left|W_{2}\left(t^{\prime}\right)-\tilde{W}_{2}\left(t^{\prime}\right)\right| .
\end{aligned}
$$

Thus

$$
\left|I_{1}\right| \leq 2 \varepsilon\left|\mu^{\prime \prime}\right|_{\text {Lip }}(\delta+\varepsilon R)^{2} \int_{0}^{t}\left|W_{2}\left(t^{\prime}\right)-\tilde{W}_{2}\left(t^{\prime}\right)\right| d t^{\prime}
$$

For the term $I_{2}$,

$$
\begin{aligned}
& \left|I_{2}\right| \leq\left|\mu^{\prime \prime}\right|_{L^{\infty}} \int_{0}^{t} \varepsilon^{1 / 2} p_{\varepsilon}^{-1}\left|W_{2}-\tilde{W}_{2}\right| \times\left|2 Z_{1}\left(t^{\prime}\right)+\varepsilon^{1 / 2} p_{\varepsilon}^{-1}\left(W_{2}+\tilde{W}_{2}\right)\right| d t^{\prime} \\
& \leq 2\left|\mu^{\prime \prime}\right|_{L^{\infty}}(\delta+\varepsilon R) \int_{0}^{t}\left|W_{2}-\tilde{W}_{2}\right| d t^{\prime} .
\end{aligned}
$$

Therefore, assuming $\delta+\varepsilon R<1$,

$$
\left|F\left(W_{2}\right)-F\left(\tilde{W}_{2}\right)\right| \leq 4\left|\mu^{\prime \prime}\right|_{\operatorname{Lip}}(\delta+\varepsilon R) \int_{0}^{t}\left|W_{2}\left(t^{\prime}\right)-\tilde{W}_{2}\left(t^{\prime}\right)\right| d t^{\prime} .
$$

For $\varepsilon<\varepsilon_{0}(\delta, \omega)$ sufficiently small, a contraction argument implies that $W_{2}(t)$ remains $O(1)$.

We turn to the study of the asymptotic behavior of $Z_{2}$ and in particular its contributions to the $O_{\infty}\left(\varepsilon^{2}\right)$ behavior of characteristics. The function $W_{2}$ satisfies the integral equation (4.19), which can be written as

$$
\begin{aligned}
& W_{2}(t)=-a t-\int_{0}^{t}\left(Z_{1}\left(t^{\prime}\right)^{2} \int_{0}^{1}(1-s) \mu^{\prime \prime}\left(\frac{Y+t^{\prime}}{\varepsilon}+\varepsilon^{1 / 2} s Z_{1}\left(t^{\prime}\right)\right) d s d t^{\prime}\right. \\
& \quad+o_{\infty}(1) .
\end{aligned}
$$


We will show that the integral term in $W_{2}$ is also $o_{\infty}(\varepsilon)$. Indeed,

$$
\begin{aligned}
& \int_{0}^{t} \varepsilon^{-1 / 2}\left(Z_{1}^{2} \int_{0}^{1}(1-s) \mu^{\prime \prime}\left(\frac{Y+t^{\prime}}{\varepsilon}+s \varepsilon^{1 / 2} Z_{1}\right) d s d t^{\prime}\right. \\
& =-\int_{0}^{t} \frac{d}{d t^{\prime}}\left(\frac{\varepsilon Z_{1}^{2}}{1+\varepsilon^{3 / 2} s \dot{Z}_{1}}\right) \mu^{\prime}\left(\frac{Y+t^{\prime}}{\varepsilon}+\varepsilon^{1 / 2} s Z_{1}\right) d t^{\prime} \\
& \quad+\left(\frac{\varepsilon Z_{1}^{2}(t)}{1+\varepsilon^{3 / 2} s \dot{Z}_{1}(t)}\right) \mu^{\prime}\left(\frac{Y+t}{\varepsilon}+\varepsilon^{1 / 2} s Z_{1}(t)\right) .
\end{aligned}
$$

In the integral we see that

$$
\frac{d}{d t^{\prime}}\left(\frac{\varepsilon Z_{1}^{2}}{1+\varepsilon^{3 / 2} s \dot{Z}_{1}}\right)=\frac{2 \varepsilon Z_{1} \dot{Z}_{1}}{1+\varepsilon^{3 / 2} s \dot{Z}_{1}}-\frac{\varepsilon Z_{1}^{2} \varepsilon^{3 / 2} s \ddot{Z}_{1}}{\left(1+\varepsilon^{3 / 2} s \dot{Z}_{1}\right)^{2}}=o_{\infty}(1)
$$

since $\dot{Z}_{1}=-\varepsilon^{-1 / 2} \mu\left(\frac{Y+t}{\varepsilon}\right)-\mu^{\prime}\left(\frac{Y+t}{\varepsilon}\right)$ and $\varepsilon^{1 / 2} Z_{1}=o_{\infty}(1)$ by Corollary 2.3 , The boundary term of (4.28) is also $o_{\infty}(1)$.

We have thus obtained that the principal contribution is simply $W_{2}(t)=-a t+o_{\infty}(1)$. Because of the nature of the integrating factor $p_{\varepsilon}$, this implies

$$
Z_{2}(t)=-a t+o_{\infty}(1) .
$$

To express $Y=Y(t ; X)$, we observe that by following the trajectories backwards, $Y$ satisfies

$$
\frac{d Y}{d s}=-c_{\varepsilon}(X, \omega):=-\left(1-\varepsilon \mu\left(\frac{Y}{\varepsilon}\right)-\varepsilon^{2} a\right), \quad s<t, \quad Y(t ; X)=X .
$$

Thus the formula (4.3) is obtained by replacing in (4.2) $t$ by $-t, t^{\prime}$ by $t-t^{\prime}$ and $\mu$ by $-\mu$.

We also need to evaluate the Jacobian $\partial_{Y} X$ and the second derivative of the characteristic transformation.

ThEOREM 4.2. The Jacobian of the transformation to characteristic coordinates is given by

$$
\partial_{X} Y=1+\varepsilon\left[\mu\left(\frac{X}{\varepsilon}\right)-\mu\left(\frac{X-t}{\varepsilon}\right)\right]+o_{\infty}(\varepsilon) .
$$

We also have

$$
\partial_{X}^{2} Y=\mu^{\prime}\left(\frac{X}{\varepsilon}\right)-\mu^{\prime}\left(\frac{X-t}{\varepsilon}\right)+o_{\infty}(1) .
$$

Proof. The Jacobian is expressed as

$$
\partial_{Y} X=1+\varepsilon^{3 / 2} \partial_{Y} Z_{1}+\varepsilon^{2} \partial_{Y} Z_{2}
$$

Computing $\partial_{Y} Z_{1}$ from (4.17) leads to

$$
\partial_{Y} Z_{1}=-\varepsilon^{-1 / 2}\left[\mu\left(\frac{Y+t}{\varepsilon}\right)-\mu\left(\frac{Y}{\varepsilon}\right)\right]+\varepsilon^{-1 / 2} \mu^{\prime}\left(\frac{Y+t}{\varepsilon}\right) \int_{0}^{t} \mu\left(\frac{Y+t^{\prime}}{\varepsilon}\right) d t^{\prime}+o_{\infty}\left(\varepsilon^{-1 / 2}\right) .
$$

Corollary 2.3 ensures that the second term of the r.h.s. of (4.35) is $o_{\infty}\left(\varepsilon^{-1 / 2}\right)$. Similarly, $\partial_{Y} Z_{2}=o_{\infty}\left(\varepsilon^{-1}\right)$. The second derivative is computed likewise. 
4.2. Transformation of the evolution equations. In this section, we show that solutions of (1.3) for $q(Y, \tau)$ written in characteristic coordinates describe the asymptotic behavior as $\varepsilon \rightarrow 0$ of solutions of (1.1). For this, we write $q(Y, \tau)=\partial_{Y} Q(Y, \tau)$, which satisfies the deterministic equation

$$
\partial_{\tau} Q=-c_{1} \partial_{Y}^{3} Q-\frac{3}{2} c_{2}\left(\partial_{Y} Q\right)^{2}+b Q
$$

We assume that $Q$ has as much regularity as we need; for example, $Q$ is in $\mathcal{S}(\mathbb{R})$ or possibly it is in $H^{k}, k \geq 3$, for all $\tau$. Define $R(X, t)=Q(Y, \tau)$, where $X=X(t, Y)$ are the trajectories (4.1) with $\mu=\frac{1}{2} \operatorname{sech}\left(D_{x}\right) \beta$ and $\tau$ is the slow time scale $\tau=\varepsilon^{2} t$. We notice that the requirement that $\mu$ be a $C^{3}$ function a.e. in $\omega$ is satisfied since the process $\beta$ that describes the bottom topography is in $L^{\infty}$.

THEOREM 4.3. The function $R(X, t)$ satisfies the following asymptotic equation:

$$
\left(\partial_{t} R+c_{\varepsilon}(X) \partial_{X} R\right)+\varepsilon^{2}\left(\frac{3 c_{2}}{2}\left(\partial_{X} R\right)^{2}+c_{1} \partial_{X}^{3} R-b R\right)=o_{d}\left(\varepsilon^{2}\right) .
$$

Proof. The various terms in the equation for $Q$ are calculated as follows:

$$
\begin{aligned}
& \varepsilon^{-2} \partial_{\tau} Q=\partial_{t} R+c_{\varepsilon}(X) \partial_{X} R \\
& \partial_{Y} Q=\left(\partial_{X} Y\right)^{-1} \partial_{X} R \\
& \partial_{Y}^{2} Q=\left(\partial_{X} Y\right)^{-2} \partial_{X}^{2} R-\left(\partial_{X} Y\right)^{-3} \partial_{X}^{2} Y \partial_{X} R
\end{aligned}
$$

and finally

$$
\begin{aligned}
\partial_{Y}^{3} Q= & \left(\partial_{X} Y\right)^{-3} \partial_{X}^{3} R-3 \partial_{X}^{2} Y\left(\partial_{X} Y\right)^{-4} \partial_{X}^{2} R \\
& +3\left(\partial_{X}^{2} Y\right)^{2}\left(\partial_{X} Y\right)^{-5} \partial_{X} R-\partial_{X}^{3} Y\left(\partial_{X} Y\right)^{-4} \partial_{X} R \\
= & \partial_{X}\left[\left(\partial_{X} Y\right)^{-3} \partial_{X}^{2} R\right]+3\left(\partial_{X}^{2} Y\right)^{2}\left(\partial_{X} Y\right)^{-5} \partial_{X} R \\
& -\partial_{X}\left[\partial_{X}^{2} Y\left(\partial_{X} Y\right)^{-4} \partial_{X} R\right]-4\left(\partial_{X}^{2} Y\right)^{2}\left(\partial_{X} Y\right)^{-5} \partial_{X} R \\
& +\partial_{X}^{2} Y\left(\partial_{X} Y\right)^{-4} \partial_{X}^{2} R \\
= & \partial_{X}\left[\left(\partial_{X} Y\right)^{-3} \partial_{X}^{2} R\right]-\partial_{X}\left[\partial_{X}^{2} Y\left(\partial_{X} Y\right)^{-4} \partial_{X} R\right] \\
& -\left(\partial_{X}^{2} Y\right)^{2}\left(\partial_{X} Y\right)^{-5} \partial_{X} R+\partial_{X}^{2} Y\left(\partial_{X} Y\right)^{-4} \partial_{X}^{2} R .
\end{aligned}
$$

The equation for $R$ becomes:

$$
\begin{aligned}
\varepsilon^{-2}\left(\partial_{t} R+c_{\varepsilon}(X) \partial_{X} R\right)= & -\frac{3 c_{2}}{2}\left(\partial_{X} Y\right)^{-2}\left(\partial_{X} R\right)^{2} \\
& -c_{1}\left(\partial_{X}\left[\left(\partial_{X} Y\right)^{-3} \partial_{X}^{2} R\right]-\partial_{X}\left[\partial_{X}^{2} Y\left(\partial_{X} Y\right)^{-4} \partial_{X} R\right]\right. \\
& \left.\quad-\left(\partial_{X}^{2} Y\right)^{2}\left(\partial_{X} Y\right)^{-5} \partial_{X} R+\partial_{X}^{2} Y\left(\partial_{X} Y\right)^{-4} \partial_{X}^{2} R\right) \\
& +b R .
\end{aligned}
$$

We now evaluate the order of the various terms and determine their contributions to the equation for $R$. Terms will be evaluated under the criterion stated in Section 2.1. The analysis of the term $\left(\partial_{X} Y\right)^{-2}\left(\partial_{X} R\right)^{2}$ in the first line of (4.38) is straightforward, from (4.32) and the smoothness of $Q$ :

$$
\partial_{X} R=\partial_{Y} Q(X-t, \tau)+O_{\infty}(\varepsilon) ;
$$

thus

$$
\left(\partial_{X} Y\right)^{-2}\left(\partial_{X} R\right)^{2}=\left(\partial_{X} R\right)^{2}+O_{\infty}(\varepsilon)
$$


In order to evaluate the other terms of (4.38), we first need an estimate on $\partial_{X}^{2} R$. From (4.38),

$$
\partial_{X}^{2} R=\partial_{Y}^{2} Q\left(X-t, \varepsilon^{2} t\right)+\left(\partial_{x} \mu\left(\frac{X}{\varepsilon}\right)-\partial_{x} \mu\left(\frac{X-t}{\varepsilon}\right)\right) \partial_{X} R+O_{\infty}(\varepsilon) .
$$

Thus, the first term in the second line of (4.38), when tested with $\varphi(X, t) \in \mathcal{S}$, is

$$
\begin{gathered}
\int \partial_{X}\left(\left(\partial_{X} Y\right)^{-3} \partial_{X}^{2} R\right) \varphi d X d t=-\int\left(\partial_{X} Y\right)^{-3} \partial_{X}^{2} R \partial_{X} \varphi d X d t \\
=\int \partial_{X}^{3} R \varphi d X d t+O_{p}(\varepsilon)
\end{gathered}
$$

Similarly, the second term in the second line of (4.38) is

$$
\int-\partial_{X}\left[\partial_{X}^{2} Y\left(\partial_{X} Y\right)^{-4} \partial_{X} R\right] \varphi d X d t=\int \partial_{X}^{2} Y\left(\partial_{X} Y\right)^{-4} \partial_{X} R \partial_{X} \varphi d X d t .
$$

From (4.32) and (4.39), this term can be written as

$$
\int \partial_{X}^{2} Y \partial_{Y} Q\left(X-t, \varepsilon^{2} t\right) \partial_{X} \varphi d X d t+O_{p}(\varepsilon)
$$

which by integration by parts leads to a contribution $O_{\infty}(\varepsilon)$. To calculate the second term in the third line of (4.38), we substitute $\partial_{X}^{2} R$ in terms of $\partial_{Y}^{2} Q$ from (4.38),

$$
\partial_{X}^{2} Y\left(\partial_{X} Y\right)^{-4} \partial_{X}^{2} R=\partial_{X}^{2} Y\left(\left(\partial_{X} Y\right)^{-2} \partial_{Y}^{2} Q+\left(\partial_{X} Y\right)^{-5} \partial_{X}^{2} Y \partial_{X} R\right) .
$$

Thus the contribution of the third line of (4.38) reduces to $\partial_{X}^{2} Y\left(\partial_{X} Y\right)^{-2} \partial_{Y}^{2} Q$. Its action on a test function is

$$
\int \partial_{X}^{2} Y\left(\partial_{X} Y\right)^{-2} \partial_{Y}^{2} Q \varphi d X d t=\int \partial_{X}^{2} Y \partial_{Y}^{2} Q \varphi d X d t+O_{p}(\varepsilon)
$$

Again by integration by parts, this term is $O_{p}(\varepsilon)$. We have obtained that the equation for $R$ reads:

$$
\varepsilon^{-2}\left(\partial_{t} R+c_{\varepsilon}(X) \partial_{X} R\right)+\frac{3 c_{2}}{2}\left(\partial_{X} R\right)^{2}+c_{1} \partial_{X}^{3} R-b R=o_{d}(1) .
$$

4.3. Asymptotic description of solutions. Returning to the original variables, we define

$$
r(X, t)=\partial_{X}\left(R^{(0)}\left(X+\frac{\varepsilon}{2} \int_{(X-t)}^{X} \gamma\left(\frac{\theta}{\varepsilon}\right) d \theta+\varepsilon^{2}\left(a+\mathrm{E}\left(\gamma^{2}\right)\right) t, t\right)\right),
$$

where $R^{(0)}(X, t)=Q\left(X-t, \varepsilon^{2} t\right)$. The function $r$ is expressed in the form of the derivative of a deterministic function evaluated along a random curve.

The scattered component $s$ is obtained by integration along left-moving characteristics, which up to the order we retain at this approximation are straight lines, with a forcing term given in terms of $r(X, t)$;

$$
s(X, t)=s^{0}(X+t)+\frac{\varepsilon^{-3 / 2}}{4} \int_{X}^{X+t} \partial_{x} \gamma\left(\frac{\theta}{\varepsilon}\right) r(\theta, t+X-\theta) d \theta .
$$

As $\varepsilon \rightarrow 0$, the characteristics tend to the limiting distribution of paths,

$$
\left.X=Y+t-\frac{\varepsilon^{3 / 2} \sigma_{\beta}}{2}(B(Y+t)-B(Y))-\varepsilon^{2}\left(a+\mathrm{E}\left(\gamma^{2}\right)\right) t\right)+o_{\infty}\left(\varepsilon^{2}\right) .
$$


An expansion in powers of $\varepsilon$ describes the asymptotic limit

$$
\begin{aligned}
& r(X, t) \sim \partial_{X}\left(R^{(0)}\left(X+\frac{\varepsilon^{3 / 2} \sigma_{\beta}}{2} B_{\omega(X)}(t), t\right)\right) \\
& \quad=R_{X}^{(0)}\left(X+\frac{\varepsilon^{3 / 2} \sigma_{\beta}}{2} B_{\omega(X)}(t), t\right)\left[1+\frac{\varepsilon^{3 / 2} \sigma_{\beta}}{2} \partial_{X} B_{\omega}(t)\right]+o_{d}\left(\varepsilon^{3 / 2}\right) .
\end{aligned}
$$

That is, the solution is given by a deterministic function $R_{X}^{(0)}$ with a phase shift given by a Brownian motion at order $\varepsilon^{3 / 2}$. Additionally its amplitude is modulated by the Jacobian of the transformation to characteristic coordinates, whose asymptotic behavior is that of a white noise process. We note in particular that the phase and amplitude processes are correlated.

The scattering term $s(X, t)$ given in (4.49) consists of two components. One arises from initial data $s^{0}(X)$ propagating along the leftwards characteristics. The second component is a superposition over the leftwards characteristics of the Bragg scattering of the solution $r(X, t)$. Because of the form (4.51), in the limit of small $\varepsilon$ it suffices to consider the contribution as being $r(X, t) \sim \partial_{X} R^{(0)}(X, t)$, for which

$$
\frac{\varepsilon^{-3 / 2}}{4} \partial_{x} \gamma\left(\frac{X}{\varepsilon}\right) r(X, t) \Rightarrow \frac{\sigma_{\beta}}{4} \partial_{X}^{2} B_{\omega}(X) r(X, t) .
$$

This very singular process is to be understood as a tempered distribution. Its integral over the leftwards characteristics has some effects of smoothing, so that the second component can be rewritten in the asymptotic limit as

$$
\begin{aligned}
\frac{\varepsilon^{-3 / 2}}{4} \int_{X}^{X+t} \quad & \partial_{x} \gamma\left(\frac{\theta}{\varepsilon}\right) \partial_{X} R^{(0)}(\theta, t+X-\theta) d \theta \\
= & \frac{\varepsilon^{-1 / 2}}{4}\left[\gamma\left(\frac{X+t}{\varepsilon}\right) \partial_{X} R^{(0)}(X+t, 0)-\gamma\left(\frac{X}{\varepsilon}\right) \partial_{X} R^{(0)}(X, t)\right] \\
& -\frac{1}{2}\left[\left(\varepsilon^{1 / 2} \int_{0}^{\theta / \varepsilon} \gamma(s) d s\right) \partial_{X}^{2} R^{(0)}(\theta, t+X-\theta)\right]_{X}^{X+t} \\
& +\frac{1}{4} \int_{X}^{X+t}\left(\varepsilon^{1 / 2} \int_{0}^{\theta / \varepsilon} \gamma(s) d s\right) \partial_{\theta}^{2} \partial_{X} R^{(0)}(\theta, t+x-\theta) d \theta \\
\Rightarrow \quad & \frac{\sigma_{\beta}}{4}\left[\partial_{X} B_{\omega}(X+t) \partial_{X} R^{(0)}(X+t, 0)-\partial_{X} B_{\omega}(X) \partial_{X} R^{(0)}(X, t)\right] \\
& -\frac{\sigma_{\beta}}{2}\left[B_{\omega}(X+t) \partial_{X}^{2} R^{(0)}(X+t, 0)-B_{\omega}(X) \partial_{X}^{2} R^{(0)}(X, t)\right] \\
& +\frac{\sigma_{\beta}}{4} \int_{X}^{X+t} B_{\omega}(\theta) \partial_{\theta}^{2} \partial_{X} R^{(0)}(\theta, t+X-\theta) d \theta .
\end{aligned}
$$

As above, this quantity is understood in the sense of a tempered distribution.

5. Consistency of the reduced system. The asymptotic behavior of integrals which depend upon the random variables $\gamma(x, \omega)$ and the solutions (4.48), (4.49) can be subtle; see for example [3], sections 3 and 5 . It follows that the terms in the system of equations (3.45), (3.46) contribute at various orders in the limit of small $\varepsilon$, and not all terms contribute at the principal order. Furthermore, making an Ansatz as to the asymptotic form of the solution, based on the anticipated form of the model equations, 
has implications on these contributions. Therefore only some of them carry over into the model equations (1.1), (1.2). Our analysis of the equations for free surface water waves in this regime of study is thus completed by verifying that the solutions obtained from the model equations (1.1), (1.2) are consistent with the underlying approximation. This process entails selecting the correct values (up to $o\left(\varepsilon^{2}\right)$ adjustments) for the parameters $a$ and $b$.

5.1. Passage from system (3.45), (3.46) to (1.1), (1.2). System (3.45)-(3.46) is a complex system of equations that is derived from the equations of water waves. In the KdV regime of principally unidirectional wave motion, as we have explained above, only some of the terms of (3.45), (3.46) play a rôle. Indeed, the solutions can be described up to errors of the order $o_{d}\left(\varepsilon^{2}\right)$ by the reduced system (1.1), (1.2), whose solutions are described in section 4. That is to say, many of the terms of (3.45), (3.46), when evaluated on a solution of (1.1), (1.2) are of order $o\left(\varepsilon^{2}\right)$, after a suitable choice of the two parameters $(a, b)$. Since these parameters enter in the solution, it is a fixed point problem to select them. Namely, one should choose parameters in (1.1), (1.2) such that the asymptotics of the terms of the equations (3.45), (3.46), when evaluated on this solution, are consistent with (1.1), (1.2).

The criterion for asymptotic behavior of a term in the equations of motion is given in terms of the asymptotic description of the vector field (3.4). That is, we say that a term $A(x, t ; \varepsilon, \omega)$ of the system of equations is of order $O_{d}\left(\varepsilon^{n}\right)$ if for any space-time test function $\varphi(X, t) \in \mathcal{S}$, the family of measures $\mathrm{P}_{\varepsilon}$ induced by

$$
\varepsilon^{-n} \int A(x, t ; \varepsilon, \omega) \varphi(X, t) d X d t
$$

converges weakly to a limit $\mathrm{P}_{0}$ as $\varepsilon \rightarrow 0$.

Using the expressions (4.48) for $r$ and (4.49) for $s$ in each term of (3.45), (3.46), choosing a test function and evaluating the resulting quantity analogous to (5.1), we retain the terms which give a contribution of order at least $O_{d}\left(\varepsilon^{2}\right)$. The remaining terms are considered to be of higher order and are dropped from the model equations. Theorem 2.1 is central to this analysis, and in particular quantities such as the mean value of functionals of the stationary processes $\beta$ and $\gamma$ describe the asymptotic limit. A detailed analysis of this procedure is carried out in [3], the outcome of which is that

$$
\begin{aligned}
a & =\frac{1}{2} a_{\beta}+\frac{1}{4} \mathrm{E}\left(\gamma^{2}\right)+\frac{1}{8} \mathrm{E}\left(\left(\partial_{x} \gamma\right)^{2}\right), \\
b & =-\frac{1}{3 \times 32} \frac{7}{8} \mathrm{E}\left(\left(\partial_{x} \gamma\right)^{3}\right),
\end{aligned}
$$

where $\gamma(x)=\operatorname{sech}\left(D_{x}\right) \beta(x)$. The coefficient $b$ has an influence on the properties of attenuation of the solution, it is a measure of a certain sense of the skewness of the distribution of $\beta$, and it can take either sign. However it is zero in the common situation in which the probability measure $\mathbf{P}$ is reversible (that is, when it is invariant under the transformation $\beta(x, \omega) \mapsto \beta(-x, \omega))$.

5.2. Comparison with previous results. The criterion dictating the asymptotic behavior of terms of the PDEs of water waves with random coefficients is somewhat different in reference 3 than in the present paper. Both seek to describe the large scale behavior of solutions in a situation in which random coefficients vary on a short spatial 
scale. Both give the same principal terms, and both approaches describe subsequent correction terms of similar form. In the present paper, however, we have articulated a somewhat different criterion for the asymptotic behavior of a vector field for which a small parameter governs the oscillatory terms; this is stated in (3.4). With this we have been more successful at capturing the form of the higher order terms of the asymptotic expansion of the solution, and their behavior is consistent with the asymptotic criterion (5.1) for the error terms. The actual difference that this makes enters into equations (1.1), (1.2) through the characteristic wave speed $c_{\varepsilon}(X)=1-\frac{\varepsilon}{2} \gamma\left(\frac{X}{\varepsilon}, \omega\right)-\varepsilon^{2} a$, where $\gamma(x, \omega)=\operatorname{sech}\left(D_{x}\right) \beta(x, \omega)$ appears in the coefficients $a$ and $b$. The statistics of the limit processes remains the same.

\section{REFERENCES}

[1] Alvarez-Samaniego, B., Lannes, D., Large time existence for $3 D$ water waves and asymptotics, Invent. Math. 171 (2008), 485-541. MR2372806 (2009b:35324)

[2] Billingsley, P., Convergence of probability measures, John Wiley \& Sons, Inc., New York-LondonSydney (1968). MR0233396 (38:1718)

[3] de Bouard, A., Craig, W., Díaz-Espinosa, O., Guyenne, P., Sulem, C., Long wave expansions for water waves over random topography, Nonlinearity 21 (2008), 2143-2178. MR.2430666

[4] Chazel, F., Influence of bottom topography on long water waves, M2AN Math. Model. Numer. Anal. 41 (2007), no. 4, 771-799. MR2362914 (2008k:76017)

[5] Craig, W., Guyenne, P. and Kalisch, H., Hamiltonian long-wave expansions for free surfaces and interfaces. Commun. Pure Appl. Math. 58 (2005), 1587-1641. MR2177163 (2006i:76012)

[6] Craig, W., Guyenne, P., Nicholls, D. and Sulem, C., Hamiltonian long-wave expansions for water waves over a rough bottom. Proc. Roy. Soc. Lond. - A 461 (2005), no. 2055, 839-87. MR2121939 (2006e:76019)

[7] Craig, W., Guyenne, P. and Sulem, C., Water waves over a random bottom. J. Fluid Mech. (2008), submitted.

[8] Doukhan, P., Mixing. Properties and Examples, Lecture Notes in Statistics 85, Springer-Verlag, 1994. MR 1312160 (96b:60090)

[9] Garnier, J., Kraenkel, R. A. and Nachbin, A., Optimal Boussinesq model for shallow-water waves interacting with a microstructure, Phys. Rev. E (3) 76 (2007), 046311. MR.2365630(2008i:76022)

[10] Garnier, J., Muñoz Grajales, J. C. and Nachbin, A., Effective behavior of solitary waves over random topography, Multiscale Model. Simul. 6 (2007), 995-1025. MR2368977 (2009a:76018)

[11] Mei, C.C. and Hancock, M., Weakly nonlinear surface waves over a random seabed, J. Fluid Mech. 475 (2003), 247-268. MR.2012498 (2004h:76026)

[12] Mei, C.C. and Li, Y., Evolution of solitons over a randomly rough seabed, Phys. Rev E 70 (2004), 016302. MR 2125713(2005j:76016)

[13] Rosales, R. and Papanicolaou, G., Gravity waves in a channel with a rough bottom. Stud. Appl. Math. 68 (1983), no. 2, 89-102. MR693716 (84e:76019)

[14] Zakharov, V. E., Stability of periodic waves of finite amplitude on the surface of a deep fluid. J. Appl. Mech. Tech. Phys. 9 (1968), 1990-1994. 